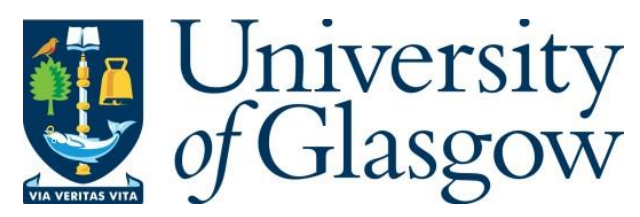

Zhang, L., Ijaz, A., Xiao, P. and Tafazolli, R. (2017) Channel equalization and interference analysis for uplink Narrowband Internet of Things (NB-IoT). IEEE Communications Letters, 21(10), pp. 2206-2209.

There may be differences between this version and the published version. You are advised to consult the publisher's version if you wish to cite from it.

http://eprints.gla.ac.uk/142609/

Deposited on: 20 June 2017

Enlighten - Research publications by members of the University of Glasgow http://eprints.gla.ac.uk 


\title{
Channel Equalization and Interference Analysis for Uplink Narrowband Internet of Things (NB-IoT)
}

\author{
Lei Zhang, Ayesha Ijaz, Pei Xiao and Rahim Tafazolli
}

\begin{abstract}
We derive the uplink system model for In-band and Guard-band narrowband Internet of Things (NB-IoT). The results reveal that the actual channel frequency response (CFR) is not a simple Fourier transform of the channel impulse response, due to sampling rate mismatch between the NB-IoT user and Long Term Evolution (LTE) base station. Consequently, a new channel equalization algorithm is proposed based on the derived effective CFR. In addition, the interference is derived analytically to facilitate the co-existence of NB-IoT and LTE signals. This work provides an example and guidance to support network slicing and service multiplexing in the physical layer.
\end{abstract}

\section{INTRODUCTION}

Narrowband Internet of Things (NB-IoT) is a sustainable technology for connecting billions of devices from a great range of utilities (e.g., smart meters, smart grid, etc), logistics (e.g., industrial assets, containers, vehicles, location and status update, etc.) to the industrial applications (process/safety monitoring, control, diagnosis report alarm, etc.) [1], [2], [3]. By using as narrow as $180 \mathrm{kHz}$ bandwidth, NB-IoT user equipment (UE) bears only $15 \%$ of complexity compared with the normal Long Term Evolution (LTE) UE; and supports up to 10 years battery longevity and $35 \mathrm{~km}$ coverage [1], [2].

To achieve the spectrum efficiency and the deployment flexibility, NB-IoT has three operation modes [1], [2]: Guardband operation that utilizes the resource blocks (RB) within the LTE carrier's guard-band; In-band operation utilizes the RB within a normal LTE carrier; Stand-alone operation utilizes any available spectrum (e.g., replacing Global System for Mobile Communications (GSM) carrier with an NB-IoT carrier). The first two modes can be deployed by reusing the LTE base station's (BS's) radio frequency (RF) front-end (i.e., the same processing bandwidth and sampling rate) and the baseband numerology (e.g., subcarrier spacing / symbol duration, frame structure ${ }^{1}$ ). This design enables the LTE BS support the two modes by software upgrade only. In this paper, we will focus on the In-band and Guard-band modes.

However, the reuse of RF and baseband processing chain in BS for NB-IoT signal may destroy the system orthogonality and invalidate the widely-used signal detection algorithms (e.g., one-tap channel equalization/estimation) for uplink transmissions [1] due to the sampling rate mismatch between the transmitter and receiver. Specifically, a much lower RF (e.g., digital to analog converter (D/A)) and baseband processing (e.g., inverse discrete Fourier transform (IDFT)) bandwidth

The authors are with the 5G Innovation Centre (5GIC) and Institute for Communication Systems (ICS), University of Surrey, Guildford, GU2 7XH, UK. The author would like to acknowledge the support of the University of Surrey 5GIC (http://www.surrey.ac.uk/5gic) members for this work.

${ }^{1}$ Note that one of the options for NB-IoT is using $3.5 \mathrm{kHz}$ subcarrier spacing to provide capacity in power-limited scenarios [1], which is different from LTE system (i.e., $15 \mathrm{kHz}$ ). However, this scenario is not within the scope of this paper. than the normal LTE system may be adopted at the NBIoT UE to reduce the complexity and energy consumption. As a result, the LTE and NB-IoT signals are multiplexed in a RF and baseband hybrid manner, instead of baseband signal multiplexing in the LTE system, where the orthogonality amongst all subcarriers and low-complexity one-tap channel equalization are secured by orthogonal frequency division multiplexing (OFDM) modulation.

For NB-IoT signal, the channel circular convolution properties and interference-free one-tap channel equalization no longer holds. Specifically, the channel impulse response (CIR) cannot be mapped to the frequency domain channel equalization coefficients by direct Fourier transform. This may invalidate the extensively used algorithms in OFDM system for channel estimation, equalization, channel state information (CSI) feedback, synchronization and system performance analysis. On the other hand, for the LTE signal, there will be interference from the NB-IoT subcarrier/symbol due to the loss of orthogonality, resulting in performance degradation. The coexistence of LTE and IoT signals has been investigated in the third generation partnership project (3GPP) study items via extensive simulations [1], with given guard-band between them to mitigate inter-service-band-interference [4]. In addition, most of the literature on NB-IoT focuses on either system frame structure design [5], random access networks [6] or scheduling [7]. However, to the best of the authors' knowledge, the analytical results on the PHY layer are still missing in the literature to provide a general guideline from the algorithm (e.g., channel estimation/equalization) design to system parameter selection (e.g., guard-band bandwidth).

In this paper, we will first establish a system model for the uplink NB-IoT system. The relationship of the CIR and the channel frequency domain equalization coefficients is derived, and one-tap channel equalization algorithm is proposed based on the derived channel model. We also prove that the IoT UE is free of interference from the LTE UE. For the LTE UE, we derive the analytical interference term that is caused by the IoT UE to guide the system design.

Notations: $\{\cdot\}^{H}$ and $\{\cdot\}^{T}$ stand for the Hermitian conjugate and transpose operation, respectively. We use $\mathbb{E}\{\mathbf{A}\}$ and $\operatorname{diag}\{\mathbf{A}\}$ to denote the expectation of matrix $\mathbf{A}$ and a diagonal matrix formed by taking the diagonal elements of $\mathbf{A}$, respectively. However, $\operatorname{diag}\{\mathbf{a}\}$ denotes a diagonal matrix formed by the vector a. $\mathbf{I}_{M}$ and $\mathbf{0}_{M \times N}$ refer to $M$ dimension identity matrix and an $M \times N$ zero matrix, respectively.

\section{NB-IOT AND LTE COEXISTING SYSTEM MODEL}

1) System descriptions and assumptions: The uplink Inband and Guard-band NB-IoT and LTE coexisting system is shown in Fig. 1. Since the operating bandwidth and sampling rate are different at the LTE UE (or BS) and NB-IoT UE, we use two sets of parameters with subscript $\{\cdot\}_{H}$ and $\{\cdot\}_{L}$ for 


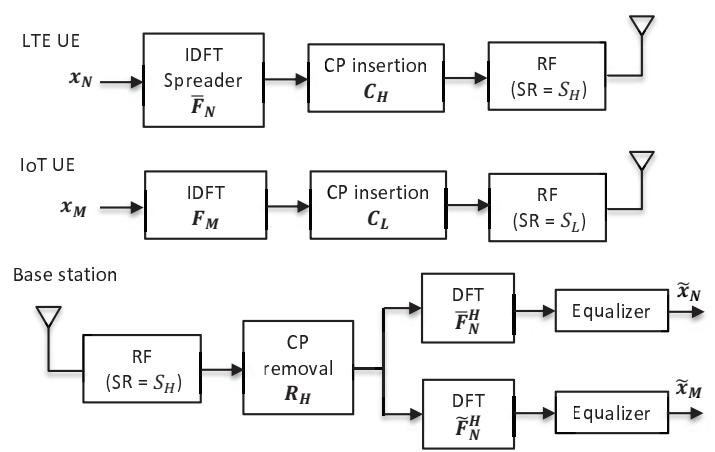

Fig. 1. Uplink NB-IoT system. (We consider one NB-IoT UE and one LTE UE, each having one RB for simplicity. Note we use two DFTs in BS for easy understanding, it can be replaced by one and takes the corresponding DFT output columns as the two UEs equalizer input. SR means sampling rate.)

high sampling rate LTE $U E$ and low sampling rate $N B-I o T$ $U E$, respectively. In Fig. 1, the IoT UE RF sampling rate $\left(S_{L}\right)$ could be as small as the IoT bandwidth (e.g., $180 \mathrm{kHz}$ ) to reduce the complexity, cost and energy assumption; while the LTE UE follows the normal LTE RF and baseband processing ${ }^{2}$. At the BS, a unified RF (with sampling rate $S_{H}$ larger than the whole system bandwidth, e.g., $30.72 \mathrm{MHz}$ ) and baseband processing is utilized to de-multiplex the two service signals.

Note that in the following we will parameterize all of the parameters, to make the derivations generally applicable to any system having mismatched sampling rate between transmitter and receiver, with the 3GPP NB-IoT system as an example. However, to simplify our derivations and without loss of generality, we assume two RBs are scheduled. One is allocated to NB-IoT UE and the other is used for LTE UE.

Let us consider that the LTE system contains overall $N$ subcarriers (i.e., DFT size is $N$ ) and each RB contains $M$ contiguous subcarriers. The DFT size for the NB-IoT system is $N_{L}=N / G \in \mathbb{R}^{+}$with $G$ being the down sampling factor. In principle, we have to set $N_{L} \geq M$ to avoid signal aliasing. To simplify our derivation and focus on the mathematical analysis, we set $N_{L}=M$ and therefore, $N=G M$. However, the derivations in this paper are applicable to $N_{L}>M$ case 3 .

We define $T_{c}$ as the symbol duration in seconds, then the sampling duration at the BS and LTE UE is $T_{s, H}=T_{c} / N$. The sampling duration for the NB-IoT will be much longer, which is $T_{s, L}=T_{c} / M$. Then the system sampling rates for LTE UE and NB-IoT device are $S_{H}=N / T_{c}$ and $S_{L}=M / T_{c}$, respectively.

2) Uplink system model: Let us write the $M$-length modulated signals for IoT and LTE services as $\mathbf{x}_{L}$ and $\mathbf{x}_{H}$, respectively. The subcarrier indexes for the two UEs are $\mathcal{U}_{H}=\left\{M_{H}, M_{H}+1, \cdots, M_{H}+M-1\right\}$ and $\mathcal{U}_{L}=$ $\left\{M_{L}, M_{L}+1, \cdots, M_{L}+M-1\right\}$, respectively, where $M_{H}$ and $M_{L}$ are the indexes of the first subcarrier for LTE and IoT UEs, respectively. The two sets are not overlapped, i.e., $\mathcal{U}_{H} \cap \mathcal{U}_{L}=\emptyset$. Then the guard band (number of subcarriers) between them is:

$$
B_{G}=\left|M_{H}-M_{L}\right|-M-1 \geq 0 .
$$

\footnotetext{
${ }^{2}$ Note that the peak-to-average-power-ratio (PAPR) reduction techniques such as DFT spreading is not considered in the paper for brevity. However it is straightforward to extend this work to the DFT spread OFDM systems.

${ }^{3}$ Indeed, when $N_{L}>M$, the unused subcarriers will be served as guardband and the processing will involve mathematically trivial subcarrier index mapping and selection.
}

After the IFFT operation, the signals at IoT and LTE UEs are $\mathbf{F}_{M} \mathbf{x}_{L}$ and $\overline{\mathbf{F}}_{N} \mathbf{x}_{H}$, respectively, where $\mathbf{F}_{M} \in \mathbb{C}^{M \times M}$ is power normalized $M$-point IDFT matrix and $\overline{\mathbf{F}}_{N} \in \mathbb{C}^{N \times M}$ is a sub-matrix of power normalized $N$-point IDFT matrix taking the corresponding $M$ columns $\in \mathcal{U}_{H}$.

Let us assume the cyclic prefix (CP) length for both UEs is $L_{C P, H}$ samples with the sample duration of $T_{s, H}$. However, according to $T_{s, L}=G T_{s, H}$, the $\mathrm{CP}$ contains $L_{C P, L}=L_{C P} / G$ NB-IoT samples (e.g., $\left.T_{s, L}\right)^{4}$. To avoid inter-symbol-interference, we assume the $\mathrm{CP}$ is longer than the channel length for both UEs. Then we can write the $\mathrm{CP}$ insertion matrices for the IoT and LTE UEs: $\mathbf{C}_{L}=$ $\left[\mathbf{0}_{L_{C P, L} \times\left(M-L_{C P, L}\right)}, \mathbf{I}_{L_{C P, L}} ; \mathbf{I}_{M}\right] \in \mathbb{R}^{\left(M+L_{C P, L}\right) \times M}$ and $\mathbf{C}_{H}=\left[\mathbf{0}_{L_{C P, H} \times\left(N-L_{C P, H}\right)}, \mathbf{I}_{L_{C P, H}} ; \mathbf{I}_{N}\right] \in \mathbb{R}^{\left(N+L_{C P, L}\right) \times N}$, respectively. After the $\mathrm{CP}$ insertion, we can represent the signals for two UEs as $\mathbf{C}_{L} \mathbf{F}_{M} \mathbf{x}_{L}$ and $\mathbf{C}_{H} \overline{\mathbf{F}}_{N} \mathbf{x}_{H}$. They have the same duration in seconds but not in number of samples.

Let us denote the multi-path CIR from IoT and LTE UEs to the $\mathrm{BS}$ as $\mathbf{h}_{L}=\left[h_{L}(1), h_{L}(2), \cdots, h_{L}\left(L_{C H, L}\right)\right]$ and $\mathbf{h}_{H}=$ $\left[h_{H}(1), h_{H}(2), \cdots, h_{H}\left(L_{C H, H}\right)\right]$, where $L_{C H, L}$ and $L_{C H, H}$ are channel lengths for $\mathbf{h}_{L}$ and $\mathbf{h}_{H}$, respectively. Without loss of generality, we assume the channel powers are normalized, i.e., $\mathbb{E}\left\{\sum_{i=1}^{L_{C H, L}}\left|h_{L}(i)\right|^{2}\right\}=1$ and $\mathbb{E}\left\{\sum_{i=1}^{L_{C H} H}\left|h_{H}(i)\right|^{2}\right\}=1$.

In the digital domain at the receiver, the BS processes the two services signals using the same baseband processing chain, thus the channel resolution is the same as the BS sampling rate and the channel length $L_{C H, L}$ is not necessarily smaller than $L_{C H, H}$. The low sampling rate IoT signal $\mathbf{C}_{L} \mathbf{F}_{M} \mathbf{x}_{L}$ will be first up-sampled by a factor of $G$ and then convolved by the multi-path channel. Equivalently, the signal will be multiplied by an up-sampling matrix $\mathbf{U}$ and then by the channel matrix $\mathbf{A}_{L}$. In this case, the number of rows in $\mathbf{A}_{L} \mathbf{U} \mathbf{C}_{L} \mathbf{F}_{M} \mathbf{x}_{L}$ for IoT UE is the same as $\mathbf{A}_{H} \mathbf{C}_{H} \overline{\mathbf{F}}_{N} \mathbf{x}_{H}$ for LTE UE. The Toeplitz channel matrices $\mathbf{A}_{L}$ and $\mathbf{A}_{H}$ are derived from their CIR with their first columns being $\left[\mathbf{h}_{L}, \mathbf{0}_{1 \times\left(N+L_{C P, H}-L_{C H, L}-1\right)}\right]^{T}$ and $\left[\mathbf{h}_{H}, \mathbf{0}_{1 \times\left(N+L_{C P, H}-L_{C H, H}-1\right)}\right]^{T}$, respectively; and their first rows being $\left[h_{L}(1), \mathbf{0}_{1 \times\left(N+L_{C P, H}-1\right)}\right]$ and $\left[h_{H}(1), \mathbf{0}_{1 \times\left(N+L_{C P, H}-1\right)}\right.$, respectively.

Consequently, the signals after $\mathrm{CP}$ removal and DFT processing at the BS for IoT (i.e., $\mathbf{y}_{L}$ ) and LTE (i.e., $\mathbf{y}_{H}$ ) UEs can be represented respectively as

$$
\begin{aligned}
\mathbf{y}_{L} & =\tilde{\mathbf{F}}_{N}^{H} \mathbf{R}_{H} \mathbf{\Psi}_{L} \mathbf{A}_{L} \mathbf{U} \mathbf{C}_{L} \mathbf{F}_{M} \mathbf{x}_{L}+\mathbf{v}_{L}+\mathbf{n}_{L} \\
\mathbf{y}_{H} & =\overline{\mathbf{F}}_{N}^{H} \mathbf{R}_{H} \mathbf{A}_{H} \mathbf{C}_{H} \overline{\mathbf{F}}_{N} \mathbf{x}_{H}+\mathbf{v}_{H}+\mathbf{n}_{H} .
\end{aligned}
$$

In equations (2) and (3), the first terms are the desired signal for IoT and LTE UEs respectively, where $\boldsymbol{\Psi}_{L}$ is the shifted carrier frequency $(\mathrm{CF})$ in the IoT device after $\mathrm{CF}$ down conversion. It is an $N+L_{C P, H}$ dimension diagonal matrix with its $i$-th diagonal element being $e^{-j 2 \pi\left(i-L_{C P, H}-1\right) M_{L} / N}$. $\mathbf{R}_{H}=\left[\mathbf{0}_{N \times L_{C P, H}}, \mathbf{I}_{N}\right]$ is the matrix for CP removal. $\tilde{\mathbf{F}}_{N}^{H} \in$ $\mathbb{C}^{M \times N}$ is a matrix that takes the $M$ rows $\in \mathcal{U}_{L}$ of the power normalized submatrix of $N$-point DFT matrix. $\mathbf{n}_{L}$ and $\mathbf{n}_{H}$ are noise vectors for IoT and LTE UE signals, respectively.

The second terms in (2) and (3) are the interference due to non-orthogonality between the two services, and

$$
\mathbf{v}_{L}=\sqrt{\rho} \tilde{\mathbf{F}}_{N}^{H} \mathbf{R}_{H} \mathbf{A}_{H} \mathbf{C}_{H} \overline{\mathbf{F}}_{N} \mathbf{x}_{H}
$$

${ }^{4}$ In this paper, we assume $L_{C P, L}=L_{C P, H} / G$ is a non-negative integer to simplify the derivations. However, when the CP length takes non-integer numbers of NB-IoT UE sampling duration, the derivations are still valid but mathematically trivial. 
is the interference from LTE RB to IoT RB, and

$$
\mathbf{v}_{H}=\frac{1}{\sqrt{\rho}} \overline{\mathbf{F}}_{N}^{H} \mathbf{R}_{H} \boldsymbol{\Psi}_{L} \mathbf{A}_{L} \mathbf{U} \mathbf{C}_{L} \mathbf{F}_{M} \mathbf{x}_{L}
$$

is the interference from IoT RB to LTE RB. Note that we defined a new parameter $\rho$ to measure the power gain difference between LTE and NB-IoT UEs, which is a compound factor of channel and transmission power gains. Specifically, the received signal power for LTE UE is $\rho$ times stronger than the one for NB-IoT.

\section{Channel Equalization And Interference ANALYSIS}

Let us define the channel frequency response from the IoT UE and the LTE UE to the BS in the diagonal matrix form as:

$$
\mathbf{H}_{L}=\operatorname{diag}\left(\hat{\mathbf{F}}_{N}^{H} \tilde{\mathbf{h}}_{L}\right), \quad \text { and } \quad \mathbf{H}_{H}=\operatorname{diag}\left(\overline{\mathbf{F}}_{N}^{H} \tilde{\mathbf{h}}_{H}\right),
$$

where $\tilde{\mathbf{h}}_{L}=\left[\mathbf{h}_{L}, \mathbf{0}_{1 \times\left(N-L_{C H, L}\right)}\right]^{T}$ and $\tilde{\mathbf{h}}_{H}=$ $\left[\mathbf{h}_{H}, \mathbf{0}_{1 \times\left(N-L_{C H, H}\right)}\right]^{T}$. $\hat{\mathbf{F}}_{N}^{H}$ is a matrix taking the first $M$ rows of $\mathbf{F}_{N}^{H}$. In addition, we define $M$-dimension diagonal matrices $\boldsymbol{\Upsilon}_{L}$ and $\boldsymbol{\Psi}_{H}$

$\boldsymbol{\Upsilon}_{L}=\frac{1}{\sqrt{G}}\left(\sum_{i=1}^{G} \boldsymbol{\Phi}_{i}\right)$, and $\boldsymbol{\Psi}_{H}=\frac{1}{\sqrt{G}} \sum_{i=0}^{G-1} e^{\frac{-j 2 \pi i\left(M+B_{G}\right)}{N}} \boldsymbol{\Phi}_{i}$,

where the $k$-th diagonal element of $\boldsymbol{\Phi}_{i}$ is $e^{-j 2 \pi i(k-1) / N}$. We have the following Theorem:

Theorem 1: Consider an uplink In-band or Guard-band NB-IoT system as shown in Fig. 1. The desired signal for the NB-IoT UE in equation (2) can be written as:

$$
\mathbf{y}_{L}=\boldsymbol{\Lambda}_{L} \mathbf{x}_{L}+\mathbf{n}_{L}=\mathbf{H}_{L} \boldsymbol{\Upsilon}_{L} \mathbf{x}_{L}+\mathbf{n}_{L},
$$

where the effective channel coefficients $\boldsymbol{\Lambda}_{L}=\mathbf{H}_{L} \boldsymbol{\Upsilon}_{L}$; and the interference from LTE to IoT UE is zero, i.e.,

$$
\mathbf{v}_{L}=\mathbf{0} .
$$

For the LTE UE, the signal model in equation (3) can be written as

$$
\mathbf{y}_{H}=\mathbf{H}_{H} \mathbf{x}_{H}+\mathbf{v}_{H}+\mathbf{n}_{H} .
$$

The power of the interference term $\mathbf{v}_{H}$ can be written as

$$
\mathbf{p}_{H}=\rho \operatorname{diag}\left(\boldsymbol{\Psi}_{H} \boldsymbol{\Psi}_{H}^{H}\right) .
$$

Proof: Let us first prove (8) and (9). Based on IoT UE signal model (2) and the relationships of $\mathbf{C}_{H}$ and $\mathbf{C}_{L}$ and $L_{C P, H}=G L_{C H, L}$, we can reform $\mathbf{R}_{H} \mathbf{\Psi}_{L} \mathbf{A}_{L} \mathbf{U C}_{L}=$ $\overline{\mathbf{\Psi}}_{L} \mathbf{R}_{H} \mathbf{A}_{L} \mathbf{U C}_{L}=\overline{\mathbf{\Psi}}_{L} \mathbf{R}_{H} \mathbf{A}_{L} \mathbf{C}_{H} \mathbf{U}=\overline{\mathbf{\Psi}}_{L} \mathbf{A}_{c i r, L} \mathbf{U}$, where $\overline{\mathbf{\Psi}}_{L}$ is a matrix comprising of the last $N$ columns and rows of $\boldsymbol{\Psi}_{L}$. In addition, we have used the fact that sufficient $\mathrm{CP}$ insertion and removal will convert the channel matrix from a Toeplitz matrix to a circular one, i.e., $\mathbf{A}_{c i r, L}=$ $\mathbf{R}_{H} \mathbf{A}_{L} \mathbf{C}_{H}$. Therefore, the first term of equation (2) (let us define it as $\left.\mathbf{y}_{L, d e s}\right)$ that contains the desired signal of IoT UE can be written as $\mathbf{y}_{L, d e s}=\tilde{\mathbf{F}}_{N}^{H} \overline{\mathbf{\Psi}}_{L} \mathbf{A}_{c i r, L} \mathbf{U} \mathbf{F}_{M} \mathbf{x}_{L}=$ $\hat{\mathbf{F}}_{N}^{H} \mathbf{A}_{c i r, L} \mathbf{F}_{N} \mathbf{F}_{N}^{H} \mathbf{U} \mathbf{F}_{M} \mathbf{x}_{L}=\mathbf{H}_{L} \hat{\mathbf{F}}_{N}^{H} \mathbf{U} \mathbf{F}_{M} \mathbf{x}_{L}=\mathbf{H}_{L}$. $\frac{1}{\sqrt{G}} \sum_{i=1}^{G} \hat{\mathbf{F}}_{M, i}^{H} \mathbf{F}_{M} \mathbf{x}_{L}$. Note that $\hat{\mathbf{F}}_{M, i}^{H}$ is a sub-matrix of $\hat{\mathbf{F}}_{N}^{H}$ taking every $M$-th column of $\hat{\mathbf{F}}_{N}^{H}$, starting from the $i$-th column, i.e, taking the $i$-th, $(i+M)$-th, $\cdots,[i+(G-1) M]$-th column of $\hat{\mathbf{F}}_{N}^{H}$. By using $\hat{\mathbf{F}}_{M, i}^{H}=\boldsymbol{\Phi}_{i} \mathbf{F}_{M}^{H}$, we have

$$
\mathbf{y}_{L, \text { des }}=1 / \sqrt{G} \cdot \mathbf{H}_{L} \sum_{i=1}^{G} \boldsymbol{\Phi}_{i} \mathbf{x}_{L}=\mathbf{H}_{L} \boldsymbol{\Upsilon}_{L} \mathbf{x}_{L}=\boldsymbol{\Lambda}_{L} \mathbf{x}_{L} .
$$

For the interference $\mathbf{v}_{L}$ from the LTE RB to IoT RB, since the BS uses standard OFDM implementation to de-multiplex IoT signal, all subcarriers sent from LTE UE are orthogonal to the subcarriers assigned to the IoT RB. i.e., $\mathbf{v}_{L}=\mathbf{0}$. Based on (12), we obtain (8).

Now let us prove (10) and (11). For the power of the interference to LTE UE, $\mathbf{p}_{H}=\mathbb{E}\left[\operatorname{diag}\left(\mathbf{v}_{H} \mathbf{v}_{H}^{H}\right)\right]$. By using (5) and following the same method that has been used to prove (8), we have

$$
\begin{aligned}
& \mathbf{p}_{H}=\rho \mathbb{E}\left[\operatorname { d i a g } \left(\tilde{\mathbf{F}}_{N}^{H} \mathbf{A}_{c i r, L} \mathbf{F}_{N} \mathbf{F}_{N}^{H} \mathbf{U} \mathbf{F}_{M} \mathbf{F}_{M}^{H} \mathbf{U}^{H} \mathbf{F}_{N} \cdot\right.\right. \\
& \left.\left.\mathbf{F}_{N}^{H} \mathbf{A}_{c i r, L}^{H} \tilde{\mathbf{F}}_{N}\right)\right]=\rho \mathbb{E}\left[\operatorname{diag}\left(\mathbf{H}_{L} \tilde{\mathbf{F}}_{N}^{H} \mathbf{U} \mathbf{F}_{M} \mathbf{F}_{M}^{H} \mathbf{U}^{H} \tilde{\mathbf{F}}_{N} \mathbf{H}_{L}^{H}\right)\right] \\
& =\rho \mathbb{E}\left\{\operatorname{diag}\left[\mathbf{H}_{L}\left(\mathbf{\Psi}_{H} \mathbf{F}_{M}^{H} \mathbf{F}_{M}\right)\left(\mathbf{\Psi}_{H} \mathbf{F}_{M}^{H} \mathbf{F}_{M}\right)^{H} \mathbf{H}_{L}^{H}\right]\right\} .
\end{aligned}
$$

By using $\mathbf{F}_{M}^{H} \mathbf{F}_{M}=\mathbf{I}_{M}$ and assumption that channel is power normalized, i.e., $\mathbb{E}\left(\mathbf{H}_{L} \mathbf{H}_{L}^{H}\right)=\mathbf{I}_{M}$, we get (11).

For LTE UE desired signal $\mathbf{y}_{H, d e s}=\overline{\mathbf{F}}_{N}^{H} \mathbf{R}_{H} \mathbf{A}_{H} \mathbf{C}_{H} \overline{\mathbf{F}}_{N} \mathbf{x}_{H}$, since it follows the standard LTE processing and satisfies the channel circular convolution property, we can derive $\mathbf{y}_{H, \text { des }}=$ $\mathbf{H}_{H} \mathbf{x}_{H}$. Substituting it into (3), we have (10).

For the Theorem 1, we have the following remarks:

Remark 1: Equation (8) reveals that the point-wise multiplication between $\Lambda_{L}$ and signal (i.e., $\mathbf{x}_{L}$ ) enables interferencefree one-tap channel frequency domain equalization. However, the equalization coefficients $\boldsymbol{\Lambda}_{L}$ for NB-IoT are not equal to the Fourier transform of CIR (i.e., $\mathbf{H}_{L}$ ). In fact, the effective channel coefficients are phase-shifted combinations of CIR DFT transform (i.e., $\mathbf{H}_{L} \boldsymbol{\Upsilon}_{L}$ ). The conclusions also imply that the original extensively used algorithms in CP-OFDM system for pilot design, channel estimation, interpolation, equalization, synchronization and system performance analysis may be no longer applicable to NB-IoT.

Remark 2: Due to the fact that the diagonal elements in $\Upsilon_{L}$ might be different, the performance of IoT UE may degrade. To improve the performance, a diagonal power compensation matrix $\mathbf{P}_{\text {com }}=\left(\boldsymbol{\Upsilon}_{L}\right)^{-1} / \eta$ can be used by precoding the transmitting signal $\mathbf{x}_{L}$ to pre-cancel the unevenly allocated power amongst the subcarriers, with $\eta$ being the normalization factor. In this case, the precoded signal $\tilde{\mathbf{x}}_{L}=\mathbf{P}_{\text {com }} \mathbf{x}_{L}$ instead of the original signal $\mathbf{x}_{L}$ will be transmitted. At the receiver side, the signal model for the IoT UE will be replaced by: $\tilde{\mathbf{y}}_{L}=1 / \eta \cdot \mathbf{H}_{L} \mathbf{x}_{L}+\mathbf{n}_{L}$.

Remark 3: For the LTE UE signal detection, equation (10) shows that the performance may be degraded by the interference $\mathbf{v}_{H}$ from NB-IoT signal, due to the NB-IoT sampling rate mismatch. The interference level depends on two factors: the power attenuation factor $\rho$ and guard-band $B_{G}$ between the LTE and NB-IoT signals. $\rho$ could be much smaller than 1 since the NB-IoT device is transmission power limited and more penetration loss could be expected since the IoT devices may be installed in extreme environment (e.g., basement). On the other hand, the impact of $B_{G}$ is shown in $\boldsymbol{\Psi}_{H}$ in (7). Relatively larger $B_{G}$ makes smaller $\left|\boldsymbol{\Psi}_{H}\right|$ and analytical results in Section IV will show the performance improvement with increasing $B_{G}$.

Remark 4: Fundamentally, the interference level on LTE UE depends on the OFDM out of band emission (OoBE). New low OoBE waveform can be implemented on the top of desired subcarrier (or RB) to attenuate the interference leakage from IoT UE to improve LTE UE performance [4]. 
Remark 5: The design is specifically for the In-band and Guard-band NB-IoT system. However, the idea is generally applicable to multi-service systems with each service having different RF/baseband bandwidth and sampling rate. The work presented in this paper provides an example and guidance to design an integrated 5G system to support network slicing and service multiplexing in the physical layer [8]. In addition, multiple NB-IoT chunks can be aggregated to support massive IoT devices, while keeping the proposed channel equalization method unaltered, but may further degrade the LTE UE performance since each chunk will create interference and will be summed up with different weight.

\section{NUMERICAL RESULTS}

In this section, we investigate the proposed channel equalization algorithm for uplink NB-IoT UE based on the derived signal model in (8). In addition, the derived analytical interference for LTE UE in equation (11) is compared with the simulated results. We consider $1 \mathrm{RB}$ for LTE UE and 1 RB for NB-IoT UE. Each RB contains 12 subcarriers. The DFT size $(N)$ and $C P$ length $\left(L_{C P, H}\right)$ are 600 and 50 respectively. We consider the LTE extended typical urban (ETU) channel. The modulation schemes for IoT UE and LTE UE are QPSK (quadrature phase-shift keying) and 16-QAM (quadrature amplitude modulation), respectively.

To verify the derived analytical interference power (11) on the LTE UE, we compare the analytical average mean square error (MSE) in the RB with simulation results in Fig. 2, with different guard-band and IoT UE attenuation factor $\rho$. It can be seen that in all cases, the analytical results perfectly match the simulated ones. As the guard band increases, the MSE reduces first and reaches the minimum value when the guard band is around half of the total bandwidth (i.e., $B_{G} \approx 300$ subcarrier). The curves go up by further increasing the guard-band. The rationale is that 0.5 is the maximum guard-band between the two UEs. The value over 0.5 will bring the two RBs close to each other due to the circular properties of baseband signal. In addition, the observation shows that increasing $\rho$ mitigates the interference linearly, which is consistent with (11).

The bit error rate (BER) performance for both LTE and IoT devices in different guard band $B_{G}$ and attenuation factor $\rho$ are shown in Fig. 3. Since IoT UE is not sensitive to $B_{G}$ and $\rho$, only one curve is given in the figure. In addition, the power compensation method proposed in Remark 2 for IoT UE is adopted in this simulation. It can be seen from the figure that when $\rho$ or $B_{G}$ increases, the LTE UE BER performance improves. In addition, it is more sensitive to $\rho$ in the small guard band scenarios. In fact, we can treat the two cases $B_{G}=$ 12 and $B_{G}=300$ as two LTE UEs that have been allocated to the worst and the best RBs in terms of the inter-serviceband-interference by the IoT UE.

\section{CONCLUSIONS}

The work introduced in this paper establishes a framework for the uplink NB-IoT system one-tap channel equalization and interference analysis. Our derivations show that the channel equalization coefficients for NB-IoT UE is a set of phaseshifted CFRs combination. In addition, the analytical interference on the LTE UE from the NB-IoT UE is derived in terms of power attenuation factor and guard band, which can be used to guide the system design and parameter selection. All results

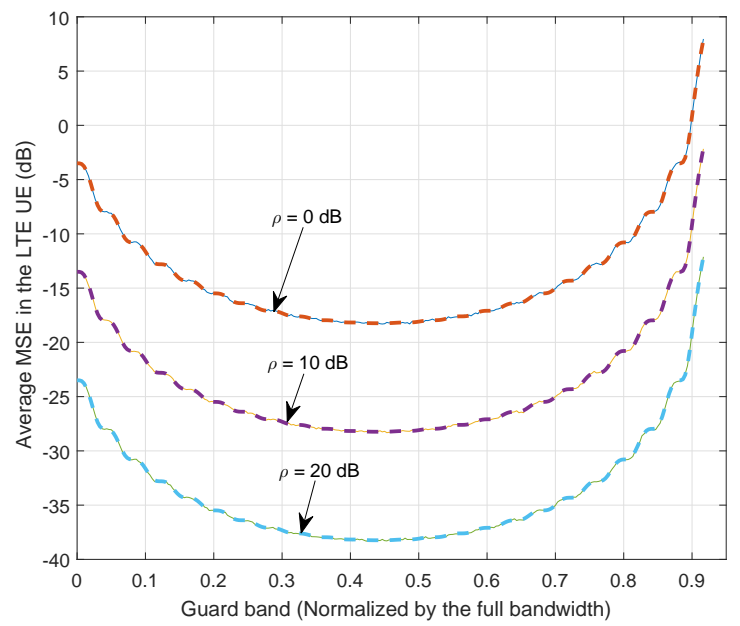

Fig. 2. LTE UE MSE versus normalized guard-band $\left(B_{G} / N\right)$ with different power attenuation factor $\rho$. (Dashed lines: Analytical results, Solid lines: Simulated results.)

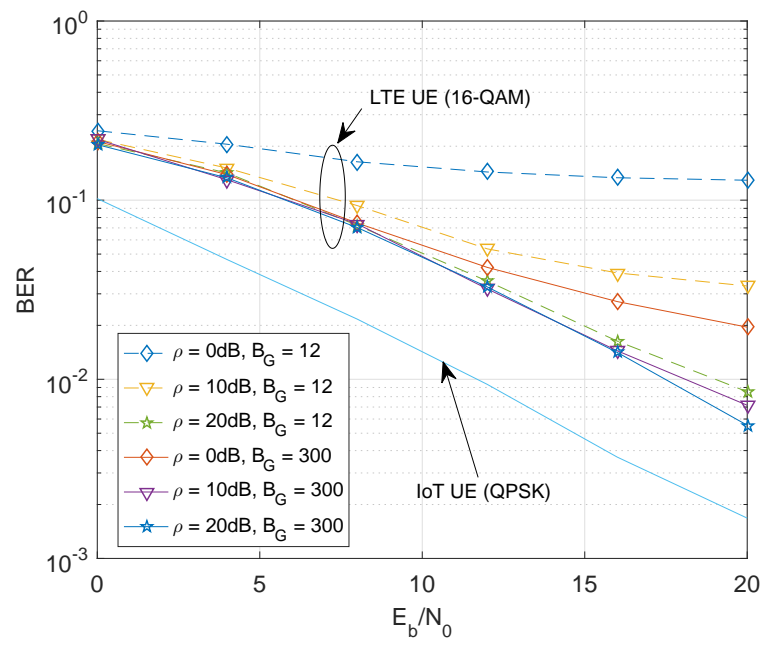

Fig. 3. BER versus $E_{b} / N_{o}$ with different power attenuation factor $\rho$ and guard band $B_{G}$.

are verified by simulations. The work presented in this paper shows an exemplary case as to how the network slicing can be underpinned in the physical layer.

\section{REFERENCES}

[1] 3GPP TR 36.802, "Narrowband Internet of Things (NB-IoT) - Technical Report for BS and UE radio transmission and reception (Release 13)," Tech. Rep., June 2016.

[2] GSMA, "3GPP Low Power Wide Area Technologies," GSMA White Paper, Tech. Rep., Oct. 2016.

[3] A. Ijaz et al., "Enabling Massive IoT in 5G and Beyond Systems: PHY Radio Frame Design Considerations," IEEE Access, vol. 4, pp. 3322 3339, 2016.

[4] L. Zhang et al., "Subband Filtered Multi-Carrier Systems for MultiService Wireless Communications," IEEE Transactions on Wireless Communications, vol. 16, no. 3, pp. 1893-1907, March 2017.

[5] Y. Wang et al., "A Primer on 3GPP Narrowband Internet of Things (NB-IoT)," [Online]. Available: https://arxiv.org/ftp/arxiv/papers/1606/1606.04171.pdf., 2016.

[6] X. Lin et al., "Random Access Preamble Design and Detection for 3GPP Narrowband IoT Systems," IEEE Wireless Communications Letters, vol. 5, no. 6, pp. 640-643, Dec 2016.

[7] C. Yu et al., "Uplink Scheduling and Link Adaptation for Narrowband Internet of Things Systems," IEEE Access, vol. PP, no. 99, pp. 1-1, 2017.

[8] L. Zhang et al., "Multi-service System: An Enabler of Flexible 5G AirInterface," IEEE Communications Magazine, pp. 1-9, 2017, to appear. 